\title{
Evolución y Tendencia de los Sistemas de Control de Motores de Combustión Interna Alternativos, una Revisión Bibliográfica
}

Evolution and Trend of Alternative Internal Combustion Motor Control Systems, A Literature Review

Trujillo-Tello J. ${ }^{1}$, Padilla-Padilla C. ${ }^{2}$, Buenaño-Moyano L. ${ }^{3}$, Cuaical-Angulo B. ${ }^{4}$
${ }^{1}$ Investigador independiente, Riobamba, Ecuador
email: juan.trujillo@ hotmail.com
${ }^{2}$ Escuela Superior Politécnica de Chimborazo, Facultad de Mecánica, Carrera de Ingeniería Automotriz, Grupo
de Investigación SAPIA, Riobamba, Ecuador
email: c_padilla@espoch.edu.ec
${ }^{3}$ Instituto Superior Tecnológico Carlos Cisneros, Carrera de Tecnología Superior en Mecánica Automotriz,
Riobamba, Ecuador
email: luis.buenano@carloscisnerosrio.onmicrosoft.com
Ecuador
emstituto Superior Tecnológico Cotopaxi, Carrera de Mantenimiento y Reparación de Motores, Latacunga,
Información del artículo
Recibido: Julio 2020
Aceptado: septiembre 2020

\section{RESUMEN}

En el presente trabajo se realiza una comparativa entre diferentes estudios relacionados a la operación de motores de combustión interna. El comportamiento del motor está regido estrictamente por los diferentes sistemas de control, entre los que se tiene a la inyección electrónica, sistemas de distribución variable, turbinas de geometría variable, entre otros. Este tipo de sistemas se encargan de realizar las diferentes adaptaciones para lograr las condiciones de funcionamiento y los requerimientos que se le exigen al motor en las diversas situaciones de conducción. La demanda de potencia, el límite de emisiones exigidas por las distintas normas, así como también la confianza y la seguridad requeridas por los usuarios de estos motores, dependen directamente del sistema de control que se haya implementado. En la actualidad, los sistemas de control electrónico se basan en el procesamiento de datos digitales y analógicos provenientes de las medidas que los distintos sensores receptan directamente del motor, y cuya información determina la activación de actuadores de acuerdo con los parámetros de funcionamiento del motor inmersos dentro de la cartografía que se ha diseñado para cada caso. Los sistemas electrónicos por su importancia y su amplio margen de control con respecto a elementos mecánicos en el motor necesitan ser calibrados y dirigidos por el software.

Palabras clave: control, sensores, calibración, tendencias, algoritmos.

\begin{abstract}
This work makes a comparison between different studies related to the operation of internal combustion engines. The behavior of the engine is strictly governed by the different control systems among which it has an electronic injection, variable distribution systems, turbines of variable geometry, among others. This type of system is in charge of making the different adaptations to achieve the operating conditions and the requirements that are demanded of the engine in various driving situations. The power demand, the emission limit required by the different standards, as well as the confidence and security required by the users of these engines, depend directly on the control system that has been implemented. Currently, electronic control systems are based on the processing of digital and analog data derived from the measurements of the different receiving sensors directly from the motor, and whose information determines the activation of the actuators according to the motor's operating parameters. immersed within the cartography that has been designed for each case. Electronic systems, due to their importance and their wide margin of control with respect to mechanical elements in the engine, need to be calibrated and directed by the software.
\end{abstract}

keywords: control, sensors, calibration, trends, algorithms. 


\section{Introducción}

Los aspectos más importantes en el sistema de control de motores de combustión interna alternativos se los determina revisando la historia de los sistemas y acciones desde los primeros procedimientos, tanto para motores de encendido por compresión (MEC), como para los motores de encendido provocado (MEP) y su evolución juntamente con los actuadores [1].

El conocimiento de los parámetros de funcionamiento de un motor conllevan al gasto, régimen, presión, temperatura, etc., son obtenidos mediante sensores de los que se analizará sus características y función, al igual que las estrategias para situaciones específicas como el arranque del motor el régimen de ralentí y sus estrategias de diagnóstico; la importancia de conocer la evolución del sistema de control está enfocada a revisar el camino que han llevado las nuevas tecnologías aplicadas en aspectos de seguridad, desempeño, costos, reducción de consumo, reducción de emisiones; la revolución de la electrónica ha cambiado la manera de controlar los parámetros de funcionamiento [2], por ello se ha considerado analizar los principios de los sistemas, su estructura, sus componentes, así como la calibración de los equipos, teniendo en cuenta las diferentes normativas anticontaminantes. Aplicando referencias, la importancia de conocer la evolución del sistema de control yace en el objetivo que tiene este sistema; en la actualidad los estándares gubernamentales limitan la producción de gases contaminantes que pueden ser producidos luego de la combustión en un motor de combustión interna alternativo (MCIA), la evolución del sistema ha podido cumplir estos estándares con eficacia ayudando así al medio ambiente. El estudio del sistema de control involucra amplios campos, desde la electrónica del vehículo [3], programas de control por software mediante algoritmos, a minuciosas investigaciones en varios campos para realizar nuevos avances; el objetivo primario de la investigación es el conocer los aspectos más relevantes que posee el sistema de control, tanto como su evolución, que surge desde los antiguos reguladores mecánicos e hidráulicos hasta los actuales sistemas de control electrónico por microprocesadores [1], cuyos sistemas pueden manipular de forma flexible los diferentes actuadores que encontramos en el motor para cumplir sus funciones y operar a fin de lograr los reque- rimientos instantáneos de potencia; además, se utilizan para la adquisición de diferentes variables de operación, tanto para cambios de temperaturas, presiones, etc., que puedan ser útiles y representativas ante la demanda de potencia del motor. Como objetivos secundarios se mostrarán las tendencias futuras del campo de control, en referencia a las propuestas actuales que brindan los elementos y sistemas que se encuentran en el motor y así generar nuevas estrategias de control que puedan implementar nuevas tecnologías con el fin de mejorar el performance, reducir consumo de combustible y, a su vez, emisiones contaminantes [4].

Los motores de combustión interna alternativos son productores de gases tóxicos enviados al medio ambiente por la fuente de energía que utilizan para su funcionamiento, que es la combustión de un hidrocarburo a base de petróleo; la reducción de estos gases ha hecho que existan normas gubernamentales a tomar en cuenta para el desarrollo de tecnologías. El principal propósito en la actualidad ya no es el encontrar el motor con mayor potencia, únicamente, sino que también se ha implementado la restricción de la contaminación que debe tener el motor, debe regirse por estas normas, mejorando así el consumo y la contaminación por la combustión del carburante con base en estas normas y en la importancia del sistema de control, ya que éste es el principal componente para regir estos parámetros. El sistema de control de motores de combustión interna alternativos [5], abarca un amplio campo de estudio, que podría iniciarse desde el análisis exhaustivo de un solo sensor para la toma exacta en tiempo reducido de un parámetro de funcionamiento de motores, tanto encendido por chispa (MEC) como por presión (MEP) de una señal sin ruido que pueda ser leída por la unidad de control electrónico y en su conjunto de un sistema de datos mediante un software diseñado para una determinada acción, éste pueda actuar de forma precisa, lo que esperamos que suceda, gracias a la mejora de su velocidad de adquisición de datos, su confiabilidad y su precisión, hasta llegar a un conjunto de sensores que conformarán un sistema aun con mayor precisión y con mayor rango de manejo de parámetros que efectuarán un funcionamiento más eficaz que producirá resultados positivos al motor tanto en su durabilidad como en su performance; aquí la importancia del estudio 
y de la historia que llevan los sistemas de control de motores de combustión interna [6].

\section{Metodología}

El método utilizado es investigativo ya que se plantean definiciones y recursos basados en varios documentos de referencia, sin excluir análisis metodológicos y prácticos para la obtención de datos aplicados a un grupo de pruebas en la toma de señales para visualizar las referencias de los valores utilizados por la unidad de control, y así definir su posible accionamiento mediante actuadores que conforman el sistema del cual se ha tomado las señales. Todo lo realizado y los adelantos desarrollados en el transcurso de las décadas, cumpliendo diferentes normas, ha sido con un propósito en común: el cuidado del medio ambiente constituye el motivo de los diferentes avances tecnológicos aplicados al sistema de control de motores.

\section{Resultados y discusión}

En la sección de resultados, un resumen de los datos recogidos y el análisis realizado sobre los más relevantes para el discurso que ha de seguir. Informar de los datos con el detalle suficiente para justificar sus conclusiones. Mencionar todos los resultados pertinentes, incluidos las que van en contra de las expectativas; asegúrese de incluir pequeños tamaños del efecto (o hallazgos estadísticamente no significativos) cuando la teoría predice que los grandes (o estadísticamente significativos). No ocultar resultados incómodos por omisión. No incluya las puntuaciones individuales o datos en bruto, con la excepción, por ejemplo, de diseños de caso único o ejemplos ilustrativos. En el espíritu de intercambio de datos (alentado por la APA y otras asociaciones profesionales $\mathrm{y}$, a veces requerida por los organismos de financiación), los datos en bruto, incluidos las características del estudio y los tamaños de los efectos individuales que se utilizan en un meta-análisis, pueden ponerse a disposición de los archivos en línea suplementarios.

\subsection{Evolución de los sistemas de control de MCIA}

Payri en su libro Motores de combustión interna alternativos da unas referencias sobre la evolución de los sistemas de control, en éste cita que desde los primeros MCIA resulta evidente la necesidad de generar y controlar los reglajes de los motores du- rante su funcionamiento; en los casos de motores por encendido provocado, aunque la potencia sea controlada mediante una válvula de mariposa, el carburador era el dispositivo capaz de dosificar de manera mayor o menor la potencia y la dosificación de combustible en función del gasto másico que entra al motor, y fue necesario el desarrollo de sistemas con mayor capacidad de adaptar el punto de encendido de la mezcla en condiciones de funcionamiento [3].

Con el fin de satisfacer y regir los requerimientos se desarrollaron sistemas centrífugos y sistemas neumáticos que adaptaban el avance de encendido respecto al régimen de giro y que aprovechaban la depreciación en el colector de admisión producido por la mariposa de admisión a bajas cargas, estos sistemas desarrollados permitían modificar la fase relativa de la leva que fijaba el ángulo de encendido para así adaptar el avance a las condiciones ideales en las que se encontraba el motor en funcionamiento [5].

Estos sistemas evolucionaron, en el sentido de la eliminación de elementos mecánicos, y surgió un mecanismo de ruptor y un sistema de generadores de impulsos inductivos y de efecto hall que ya formaron parte de componentes electrónicos para comandar las bujías [7].

Estos avances permanecieron durante años y en nuestro país siguen operando en una cantidad considerable gracias a su diseño, pero no bastó y fue complicándose en los siguientes años debido a la implementación de componentes electrónicos $\mathrm{y}$ a las normativas anticontaminantes que surgieron en el año 1980, por lo que se decidió mejorar el sistema de control para la adaptación del ralentí. Es aquí cuando el control del dosado se vuelve importante, permitiendo a los catalizadores de tres vías implementarse como nueva tecnología en MEP y requerir de un balance estequiométrico de la combustión para así reducir sus emisiones [8].

Con el tiempo se fueron presentando dificultades en el dosado estequiométrico, por lo cual surgió la necesidad de introducir nuevos sistemas para la inyección que fue controlada electrónicamente, lo que definitivamente mejoraría las prestaciones del sistema de carburación [10]. Ya que estos sistemas ofrecen un control preciso y controlado fue implementado una sonda de concentración de oxígeno en el colector de escape que se la llamó 
sonda lambda, que precisa el uso de la electrónica de inyección [11].

Con el avance y la aparición de sistemas que gestionan la inyección electrónicamente, por medio de sistemas digitales basados en microcontroladores, los sistemas del motor fueron evolucionando progresivamente hasta obtenerse un control netamente electrónico; este avance llegó al punto que el usuario no controla los elementos mecánicos, sino que el sistema de control, a partir de señales, determina las acciones que debe realizar [12].
Aunque las normativas vigentes solo mencionan la reducción de gases contaminantes no rige disposición en la implementación de sistemas electrónicos para el control de motores, sin embargo, las normativas de autodiagnóstico se pusieron como un parámetro obligado para el funcionamiento del motor que se denominó OBD (on board diagnostic) para realizar un monitoreo continuo de los sensores y sistemas para la reducción de emisiones del motor [13]. En la tabla 1 se muestra el avance y evolución de los sistemas de inyección y una breve diferencia entre cada uno de éstos.

Tabla 1. Comparación entre algunos tipos de sistemas de inyección

\begin{tabular}{|c|c|c|c|c|c|c|c|}
\hline $\begin{array}{l}\text { K-JETRO- } \\
\text { NIC }\end{array}$ & $\begin{array}{c}\text { KE-JETRON- } \\
\text { IC }\end{array}$ & $\begin{array}{c}\text { L-JETRON- } \\
\text { IC }\end{array}$ & $\begin{array}{l}\text { LE-JETRO- } \\
\text { NIC }\end{array}$ & $\begin{array}{l}\text { LE2-JE- } \\
\text { TRONIC }\end{array}$ & $\begin{array}{l}\text { LE3-JETRON- } \\
\text { IC }\end{array}$ & $\begin{array}{c}\text { MOTRON- } \\
\text { IC ML } 4.1\end{array}$ & $\begin{array}{c}\text { MOTRON- } \\
\text { IC ML } 2.5\end{array}$ \\
\hline $\begin{array}{l}\text { Inyección } \\
\text { mecánica, } \\
\text { indirecta, } \\
\text { intermitente } \\
\text { y simultánea }\end{array}$ & $\begin{array}{l}\text { Inyección } \\
\text { mixta, in- } \\
\text { directa, in- } \\
\text { termitente y } \\
\text { simultánea }\end{array}$ & $\begin{array}{l}\text { Inyección } \\
\text { mixta, in- } \\
\text { directa, in- } \\
\text { termitente y } \\
\text { simultánea }\end{array}$ & $\begin{array}{l}\text { Inyección } \\
\text { mixta, in- } \\
\text { directa, in- } \\
\text { termitente y } \\
\text { simultánea }\end{array}$ & $\begin{array}{l}\text { Inyección } \\
\text { mixta, in- } \\
\text { directa, in- } \\
\text { termitente y } \\
\text { simultánea }\end{array}$ & $\begin{array}{l}\text { Inyección } \\
\text { mixta, in- } \\
\text { directa, in- } \\
\text { termitente y } \\
\text { simultánea }\end{array}$ & $\begin{array}{l}\text { Inyección } \\
\text { mixta, in- } \\
\text { directa, in- } \\
\text { termitente y } \\
\text { simultánea }\end{array}$ & $\begin{array}{l}\text { Inyección } \\
\text { mixta, in- } \\
\text { directa, in- } \\
\text { termitente y } \\
\text { secuencial }\end{array}$ \\
\hline $\begin{array}{l}\text { Rampa de } \\
\text { inyectores } \\
\text { de sección } \\
\text { redonda }\end{array}$ & $\begin{array}{l}\text { Rampa de } \\
\text { inyectores } \\
\text { de sección } \\
\text { redonda }\end{array}$ & $\begin{array}{l}\text { Rampa de } \\
\text { inyectores } \\
\text { de sección } \\
\text { redonda }\end{array}$ & $\begin{array}{l}\text { Rampa de } \\
\text { inyectores } \\
\text { de sección } \\
\text { redonda }\end{array}$ & $\begin{array}{c}\text { Rampa de } \\
\text { inyectores de } \\
\text { sección cua- } \\
\text { drada }\end{array}$ & $\begin{array}{c}\text { Rampa de } \\
\text { inyectores de } \\
\text { sección cua- } \\
\text { drada }\end{array}$ & $\begin{array}{l}\text { Rampa de } \\
\text { inyectores de } \\
\text { sección cua- } \\
\text { drada }\end{array}$ & $\begin{array}{c}\text { Rampa de } \\
\text { inyectores de } \\
\text { sección cua- } \\
\text { drada }\end{array}$ \\
\hline $\begin{array}{c}\text { Inyectores } \\
\text { mecánicos. } \\
\text { Apertura: } 3.5 \\
\text { bar }\end{array}$ & $\begin{array}{c}\text { Inyectores } \\
\text { mecánicos. } \\
\text { Apertura: } 3.5 \\
\text { bar }\end{array}$ & $\begin{array}{l}\text { Inyectores: } \\
\text { tensión de } \\
\text { apertura } \\
3 \text { voltios } \\
(\mathrm{R}=2,4-2,6 \\
\Omega)\end{array}$ & $\begin{array}{l}\text { Inyectores: } \\
\text { tensión de } \\
\text { apertura } 12 \text {, } \\
\text { con solenoi- } \\
\text { de de latón } \\
(\mathrm{R}=14-16 \\
\Omega)\end{array}$ & $\begin{array}{c}\text { Inyectores: } \\
\text { tensión de } \\
\text { apertura } 12, \\
\text { con solenoi- } \\
\text { de de latón } \\
(\mathrm{R}=14-16 \\
\Omega)\end{array}$ & $\begin{array}{c}\text { Inyectores: } \\
\text { tensión de } \\
\text { apertura } 12, \\
\text { con solenoi- } \\
\text { de de latón } \\
(\mathrm{R}=14-16 \\
\Omega)\end{array}$ & $\begin{array}{c}\text { Inyectores: } \\
\text { tensión de } \\
\text { apertura } 12 \text {, } \\
\text { con solenoi- } \\
\text { de de latón } \\
(\mathrm{R}=14-16 \\
\Omega)\end{array}$ & $\begin{array}{l}\text { Inyectores: } \\
\text { tensión de } \\
\text { apertura } 12, \\
\text { con solenoi- } \\
\text { de de latón } \\
(\mathrm{R}=14-16 \\
\Omega)\end{array}$ \\
\hline $\begin{array}{l}\text { Medición } \\
\text { de aire por } \\
\text { plato-sonda }\end{array}$ & $\begin{array}{l}\text { Medición } \\
\text { de aire por } \\
\text { plato-sonda }\end{array}$ & $\begin{array}{l}\text { Medición } \\
\text { de aire por } \\
\text { flujómetro } \\
\text { de } 7 \text { pines }\end{array}$ & $\begin{array}{l}\text { Por flujó- } \\
\text { metro de } 5 \\
\text { pines. Aleta } \\
\text { sonda con } \\
\text { válvula de } \\
\text { seguridad }\end{array}$ & $\begin{array}{l}\text { Medición } \\
\text { de aire por } \\
\text { flujómetro } \\
\text { de } 5 \text { pines }\end{array}$ & $\begin{array}{l}\text { Medición } \\
\text { de aire por } \\
\text { flujómetro } \\
\text { de } 4 \text { pines }\end{array}$ & $\begin{array}{l}\text { Medición } \\
\text { de aire por } \\
\text { flujómetro } \\
\text { de } 5 \text { pines }\end{array}$ & $\begin{array}{l}\text { Medición de } \\
\text { aire por hilo } \\
\text { caliente }\end{array}$ \\
\hline
\end{tabular}




\begin{tabular}{|c|c|c|c|c|c|c|c|}
\hline $\begin{array}{l}\text { Relé taqui- } \\
\text { métrico }\end{array}$ & $\begin{array}{l}\text { Relé taqui- } \\
\text { métrico }\end{array}$ & $\begin{array}{c}\text { Relé doble e } \\
\text { interruptor } \\
\text { seguridad } \\
\text { electrobom- } \\
\text { ba en flujó- } \\
\text { metro }\end{array}$ & $\begin{array}{l}\text { Relé taqui- } \\
\text { métrico }\end{array}$ & $\begin{array}{l}\text { Relé taqui- } \\
\text { métrico }\end{array}$ & $\begin{array}{c}\text { interruptor } \\
\text { seguridad } \\
\text { electrobom- } \\
\text { ba por flujó- } \\
\text { metro elec- } \\
\text { trobomba } \\
\text { comandado } \\
\text { por la ECU }\end{array}$ & $\begin{array}{c}\text { interruptor } \\
\text { seguridad } \\
\text { electrobom- } \\
\text { ba por flujó- } \\
\text { metro elec- } \\
\text { trobomba } \\
\text { comandado } \\
\text { por la ECU }\end{array}$ & $\begin{array}{c}\text { interruptor } \\
\text { seguridad } \\
\text { electrobom- } \\
\text { ba por flujó- } \\
\text { metro elec- } \\
\text { trobomba } \\
\text { comandado } \\
\text { por la ECU }\end{array}$ \\
\hline $\begin{array}{l}\text { Inyector de } \\
\text { arranque en } \\
\text { frío controla- } \\
\text { do por termo } \\
\text { contacto } \\
\text { temporizado }\end{array}$ & $\begin{array}{l}\text { Inyector de } \\
\text { arranque en } \\
\text { frío controla- } \\
\text { do por termo } \\
\text { contacto } \\
\text { temporizado }\end{array}$ & $\begin{array}{l}\text { Inyector de } \\
\text { arranque en } \\
\text { frío controla- } \\
\text { do por termo } \\
\text { contacto } \\
\text { temporizado }\end{array}$ & $\begin{array}{l}\text { Inyector de } \\
\text { arranque en } \\
\text { frío controla- } \\
\text { do por termo } \\
\text { contacto } \\
\text { temporizado }\end{array}$ & $\begin{array}{c}\text { No lleva. } \\
\text { Duplicación } \\
\text { de impulsos } \\
\text { en la fase de } \\
\text { arranque en } \\
\text { frío }\end{array}$ & $\begin{array}{c}\text { No lleva. } \\
\text { Duplicación } \\
\text { de impulsos } \\
\text { en la fase de } \\
\text { arranque en } \\
\text { frío }\end{array}$ & $\begin{array}{c}\text { No lleva. } \\
\text { Duplicación } \\
\text { de impulsos } \\
\text { en la fase de } \\
\text { arranque en } \\
\text { frío }\end{array}$ & $\begin{array}{c}\text { No lleva. } \\
\text { Duplicación } \\
\text { de impulsos } \\
\text { en la fase de } \\
\text { arranque en } \\
\text { frío }\end{array}$ \\
\hline $\begin{array}{c}\text { Regulación } \\
\text { de riqueza } \\
\text { por tornillo } \\
\text { de } \\
\text { acciona- } \\
\text { miento sobre } \\
\text { plato-sonda }\end{array}$ & $\begin{array}{l}\text { Regulación } \\
\text { de riqueza } \\
\text { por tornillo } \\
\text { de acciona- } \\
\text { miento sobre } \\
\text { plato-sonda } \\
\text { y control por } \\
\text { regulador } \\
\text { electro- hi- } \\
\text { dráulico }\end{array}$ & $\begin{array}{l}\text { Regulación } \\
\text { de riqueza } \\
\text { por by pass } \\
\text { en flujóme- } \\
\quad \text { tro }\end{array}$ & $\begin{array}{l}\text { Regulación } \\
\text { de riqueza } \\
\text { por by pass } \\
\text { en flujóme- } \\
\text { tro }\end{array}$ & $\begin{array}{c}\text { Regulación } \\
\text { de riqueza } \\
\text { por by pass } \\
\text { en flujóme- } \\
\text { tro }\end{array}$ & $\begin{array}{l}\text { Potenció- } \\
\text { metro de } \\
\text { control de } \\
\text { oxígeno en el } \\
\text { flujómetro }\end{array}$ & $\begin{array}{l}\text { Potenció- } \\
\text { metro de } \\
\text { control de } \\
\text { oxígeno en el } \\
\text { flujómetro }\end{array}$ & $\begin{array}{l}\text { Potenció- } \\
\text { metro de } \\
\text { control de } \\
\text { oxígeno en el } \\
\text { medidor de } \\
\text { hilo caliente }\end{array}$ \\
\hline Sin ECU & $\begin{array}{l}\text { ECU analó- } \\
\text { gica de } 25 \\
\text { pines }\end{array}$ & $\begin{array}{c}\text { ECU analó- } \\
\text { gica de } 35 \\
\text { pines }\end{array}$ & $\begin{array}{c}\text { ECU ana- } \\
\text { lógica de } 25 \\
\text { pines }\end{array}$ & $\begin{array}{c}\text { ECU ana- } \\
\text { lógica de } 25 \\
\text { pines }\end{array}$ & $\begin{array}{l}\text { ECU digital } \\
\text { de } 15 \text { pines }\end{array}$ & $\begin{array}{l}\text { ECU digital } \\
\text { de } 35 \text { pines }\end{array}$ & $\begin{array}{l}\text { ECU digital } \\
\text { de } 55 \text { pines }\end{array}$ \\
\hline Sin ECU & Sin ECU & Sin ECU & Sin ECU & $\begin{array}{l}\text { ECU: enri- } \\
\text { quecimiento } \\
\text { en fase de } \\
\text { arranque en } \\
\text { frío y corte } \\
\text { en decelera- } \\
\text { ción. }\end{array}$ & $\begin{array}{l}\text { ECU: enri- } \\
\text { quecimiento } \\
\text { en fase de } \\
\text { arranque en } \\
\text { frío y corte } \\
\text { en decelera- } \\
\text { ción. }\end{array}$ & $\begin{array}{l}\text { ECU: enri- } \\
\text { quecimiento } \\
\text { en fase de } \\
\text { arranque en } \\
\text { frío y corte } \\
\text { en decelera- } \\
\text { ción. }\end{array}$ & $\begin{array}{l}\text { ECU: enri- } \\
\text { quecimiento } \\
\text { en fase de } \\
\text { arranque en } \\
\text { frío y corte } \\
\text { en decelera- } \\
\text { ción. }\end{array}$ \\
\hline Sin ECU & $\begin{array}{l}\text { ECU en el } \\
\text { interior del } \\
\text { vehículo }\end{array}$ & $\begin{array}{l}\text { ECU en el } \\
\text { interior del } \\
\text { vehículo }\end{array}$ & $\begin{array}{l}\text { ECU en el } \\
\text { interior del } \\
\text { vehículo }\end{array}$ & $\begin{array}{l}\text { ECU en el } \\
\text { interior del } \\
\text { vehículo }\end{array}$ & $\begin{array}{l}\text { ECU mon- } \\
\text { tada sobre el } \\
\text { flujómetro }\end{array}$ & $\begin{array}{l}\text { ECU en el } \\
\text { interior del } \\
\text { vehículo }\end{array}$ & $\begin{array}{c}\text { ECU en el } \\
\text { interior del } \\
\text { vehículo }\end{array}$ \\
\hline
\end{tabular}




\subsection{Configuración y estructura de sistemas de control}

Los avances han hecho que los sistemas de control electrónico se extiendan a todos los sistemas que contiene un automóvil; en este apartado se revisarán lo componentes del sistema de control electrónico para motores, entre los cuales tenemos: sensores, actuadores, cables, unidad de control [3].

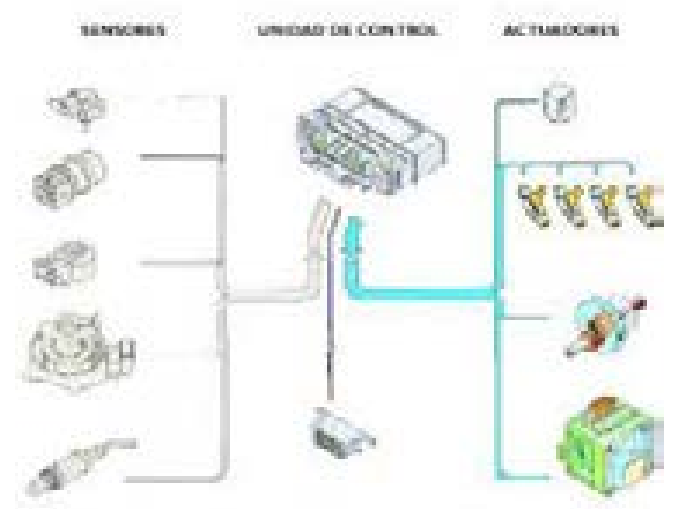

Figura 1. Principales sensores presentes en motores actuales [14].

La figura 2 muestra una unidad de control electrónico ECU, en ella se agrupan y reciben todas las señales, las etapas de conversión analógicas-digitales, la unidad de procesamiento ( $\mathrm{CPU}$ ) de datos y tiempo, las cuales permiten la sincronía con el cigüeñal, la CPU ha sufrido cambios con el tiempo pasando de la tecnología de 8 bits hasta las actuales de 32 bits. Finalmente, es el cableado el que se encarga de alimentar los sensores y actuadores y distribuir correctamente las señales que brinda la ECU, los cables necesarios pueden ser secciones considerables por la potencia eléctrica necesaria [14].

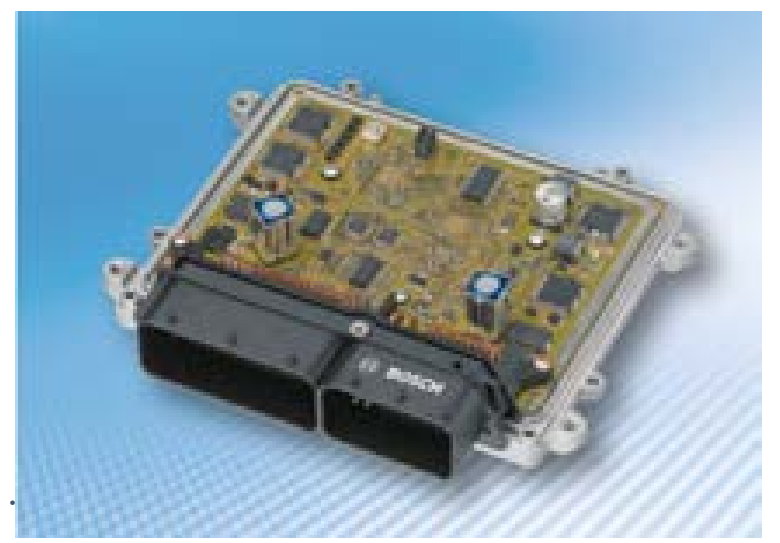

Figura 2. ECU comercial [16].
Se destaca la utilización en el mercado mundial de sensores inteligentes, los cuales, aparte de recibir la señal, poseen la conversión analógica digital y comunican estas señales a través de una red de datos a la ECU. La red o bus de datos estándar conocida como CAN (controller area network) es la más utilizada en automóviles

Los principales objetivos de esta implementación son:

- reducir el costo del cableado, ya que el único bus de datos es el encargado de intercomunicar todos los sistemas;

- permitir el intercambio de componentes; $y$,

- reducir los problemas de ruido en las señales digitales y mejorar la compatibilidad.

\subsection{Adquisición de parámetros de funcio- namiento de MCIA}

Como se ha descrito, el sistema de control requiere de la presencia de numerosos sensores disponibles en el motor para indicar el estado de un sistema en particular; aun existiendo una amplia variedad de sensores se restringen a su precio, vida útil o precisión; por este inconveniente se limita el uso y se generaliza la aplicación de una cantidad mínima de sensores en automoción. En motores de mayor precio puede permitirse el empleo de sensores más costosos y sofisticados, pero no sucede en general.

\subsection{Algoritmos del sistema de control}

El software de control tiene una estructura netamente funcional, de forma que existen algoritmos dedicados específicamente a la lectura y control de los diferentes sistemas implementados en los vehículos, este intercambio de información de componente a componente se realiza a través de varias capas lógicas superiores que los coordinan; en parte la unidad de procesado de tiempo es la que sincroniza la posición del cigüeñal de los demás sistemas que lo requieren, como el sistema de inyección de encendido.

La ECU coordina las estructuras de control empleadas en sistemas de bucle abierto y cerrado que permiten variar según el funcionamiento del motor [15]. 


\section{Bucle abierto}

Las acciones que controlan los sistemas se calculan directamente en base a medidas del motor y variables programadas, estos sistemas brindan gran estabilidad y no necesitan de sensores específicos que midan la variable a controlar; sin embargo, los sistemas accionados por control de bucle abierto son necesarios, como el control de combustible inyectado en MEC.

\section{Bucle cerrado}

Existen medidas directas de la variable que se desea controlar, en comparación con bucle abierto, este controlador permite cancelar los errores de posición que asegura que el valor final de la variable sea el deseado a costa de una mayor complejidad y riesgo de inestabilidades, ya que en los MCIA se encuentran comportamientos no lineales, que están sujetos a ser saturados; resulta necesario variar las constantes del controlador en función de los parámetros de funcionamiento o las condiciones de operación y de las magnitudes y signos de error del bucle, lo que lleva a un esfuerzo de calibración precisa.

La diferencia que existe entre el control MEP y los $\mathrm{MEC}$ rige en la diferencia de combustión y el control del sistema de admisión de aire, ya que los motores por encendido provocado necesitan rigurosamente el control del dosado estequiométrico, lo cual no ocurre en los motores por compresión.

\subsection{Control de aire}

En los motores de encendido por compresión naturalmente aspirados sin el sistema de recirculación de gases, no es necesario el control de aire, en parte, los MEC actualmente son incorporados con turbocompresores que los vuelve sobrealimentados y poseen un sistema de recirculación de gases de escape, para evitar inestabilidades entre cada sistema se ha decidido implementar un control independiente para determinadas variables necesarias.

\subsection{Inyección y sistema de limitación}

En los motores de encendido por compresión el sistema de alimentación de combustible es netamente independiente del sistema de renovación de carga cuando el motor se encuentra en condiciones estacionarias, los sistemas de control son por bucle abierto en función de la operación y de va- riables auxiliares, se selecciona la presión a la cual debe ingresar el combustible, la cantidad de inyecciones a realizarse, el instante en el cual debe producirse cada una de las inyecciones y su duración. El uso de inyecciones precargadas permite reducir enormemente el ruido, tanto que con las pos-inyecciones se controla las emisiones de partículas; la presión del sistema de inyección se controla en bucles cerrados con el fin de mantener un valor de referencia establecido inicialmente.

\subsection{Ralentí y arranque}

Estos dos estados de operación específicos encontrados en los motores son abordados por sus características particulares. En el régimen de ralentí los cambios de consigna son inferiores al 5\% de sus valores habituales, una vez encontrado el error el régimen real de funcionamiento que rige el motor actúa, y está gobernado en general por un regulador, el sistema de control de ralentí es independiente y diferente de los utilizados en el motor cuando se encuentra a plena carga y se activa solo cuando la demanda de potencia es prácticamente nula. Este control de ralentí en motores actuales ha producido una considerable disminución del combustible consumido y sobre todo en MEP la reducción de emisiones contaminantes al medio ambiente.

La operación del arranque del motor tiene una forma particular en las estrategias de control, ya que puede realizarse sin dificultad en unas situaciones y muy dificilmente en otras, por esta razón es necesario tener una estrategia específica que sea suficientemente robusta para el arranque, incluso con condiciones desfavorables, cuya acción es condicionada por la reducida potencia.

Se establece estrategias una vez que el usuario actúa sobre los sistemas de arranque:

- arranque inicial

- aceleración rápida

- final de arranque

\subsection{Diagnóstico}

Debido a la creciente preocupación por las emisiones vehiculares, el sistema del motor está obligado a realizar tareas de diagnóstico, capaces de detectar el mal funcionamiento del mismo. Los sistemas a diagnosticar son el sistema de inyección, recircu- 
lación de gases de escape, control de combustible, sobrealimentación, la eficiencia de los sistemas de postratamiento; ya que el número de funciones de diagnóstico es creciente en motores actuales surge la necesidad de incluir un determinado sensor en el sistema. Como referencia, en los motores de encendido provocado se utiliza dos sondas lamba con el fin de diagnosticar el correcto funcionamiento del catalizador, encargado de reducir los gases contaminantes por reacciones químicas, este sistema avisa al usuario si existe alguna falla mediante luces de testigo en el panel de control, y a su vez almacena esta información sobre los errores a través de un conector normalizado para facilitar el mantenimiento, según sea la gravedad de la falla la ECU adopta diversas acciones para reducir el impacto y proteger el sistema. Así, en fallos netamente riesgosos el sistema puede inmovilizar el arranque o limitar la potencia entregada en el motor [15].

\subsection{Optimización y calibración en MCIA}

Como desventaja los microcontroladores trabajan con gran cantidad de información que define el accionamiento de sistemas de control en función de datos que brindan los sensores a cada instante, el proceso que sigue para obtener las actuaciones que efectuará el motor en condiciones de operaciones se lo conoce como proceso de calibración del motor; a los parámetros de funcionamiento obtenidos durante el proceso se los denomina parámetros de calibración, entre los cuales se encuentran valores de referencia como presión de inyección, avance de inyección, gasto de aire, temperatura, etc.

La complejidad de este proceso se refleja en los aspectos a tomarse en cuenta que son:

- de la calibración que se realice depende el comportamiento, prestaciones y emisiones contaminantes del motor;

- $\quad$ el sistema puede tener calibraciones diferentes según la operación ya que es un sistema no lineal el cual dificulta en total su calibración;

- las normativas de emisiones contaminantes y la protección de la mecánica establecen límites y criterios para la calibración, y,

- la calibración realizada a los motores debe ser netamente en operaciones dinámicas ya que la variación a las que se encuentra sometido es rápida como el régimen de giro y el par, por esta razón no basta una calibración en puntos estabilizados.

La calibración se la realiza primero estáticamente y posteriormente en un banco de pruebas dinámico donde se ajustan los controladores y se prueba la dinámica del motor para validar el funcionamiento del mismo en diferentes condiciones [15].

\subsection{Tendencias del mercado y control de MCIA}

Se espera que la importancia de los sistemas de control se mantenga creciente y se refuercen con:

- incorporación de numerosos sistemas de control electrónicos;

- $\quad$ aumento en la flexibilidad del motor que sea capaz de adaptarse a distintos modos de operaciones y diferentes combustibles para aprovechar una mayor tendencia del mercado;

- aumentar la capacidad de autodiagnóstico no controlada por los usuarios;

- coordinación de diferentes parámetros y sistemas para el confort y seguridad de los usuarios;

- aumento en la coordinación inalámbrica con diferentes flotas de vehículos que rijan la gestión de tráfico mejorando la seguridad; e,

- investigación de sistemas autodirigidos controlados electrónicamente con el fin de simplificar el proceso del tráfico existente.

Existen dos líneas de evolución no excluyentes:

1. Desarrollo de sistemas de control que se basan en modelos para describir el funcionamiento del motor, los cuales pueden usarse como un modelo de predicción en un sistema de control predictivo. La principal ventaja de estos sistemas es notoria, por el empleo de modelos físicos que contienen la descripción de fenómenos que puedan surgir en el motor que va a necesitar menores parámetros experimentales. Requiere un aumento significativo de microcontroladores, así como métodos para identificar los modelos para brindar la confianza del comportamiento del motor. 
2. Sistemas que se adapten con los de control, que sean capaces de mejorar las prestaciones, basados en captadores mediante algoritmos y lenguajes de aprendizaje continuo. Este sistema permitiría absorber dispersiones y desgaste de señales para moldear los parámetros en tiempo más preciso y real.

Actualmente existe grandes limitaciones para el desarrollo netamente automático de un vehículo, por una parte, se encuentra la falta de sensores capaces de proporcionar información no supervisada con rigidez y precisión y un sistema que pueda proporcionar información sobre las emisiones contaminantes y prestaciones exactas que pueden brindar los diferente MCIA.

\section{Conclusiones}

La implementación de sistemas de control ha venido evolucionando desde la primera aplicación en los automotores con la invención de los componentes electrónicos y microprocesadores que han brindado resultados favorables para el control de los parámetros de funcionamiento de MCIA y del uso de sensores y actuadores para brindar estabilidad de motor a plena carga, en ralentí y arranque, sin despreciar la importancia que tuvieron, para su invención, evolución y funcionamiento, las normas regidas por departamentos gubernamentales para la reducción de emisiones de gases contaminantes emitidos por MCIA. En este punto, cabe recalcar la importancia de la historia y la evolución con el pasar de los tiempos para enfocarnos en tendencias futuras a fin de que se mantenga la importancia de los sistemas de control electrónico en el motor que vendrán con el avance de tecnologías aplicadas a los microprocesadores.

\section{Referencias}

[1] L. Guxxella, Introduction to modeling and control of internal combustion engine systems, Springer, 2010.

[2] R. Van Basshunyesen, Internal combustion engine handbook: basics, components, systems, and perspectives., SAE International, 2004.
[3] J. M. D. Francisco Payri, Motores de combustión interna alternativos, $1 .^{\text {a }}$ ed., vol. I, Reverte, 2011.

[4] Sciarretta, A y Guzella, L, «Control of hybrid electric vechicles,» IEEE Control Systems Magazine, pp. 60-70, 2007.

[5] J. Conesa, «Motor de Combustión Interna,» 2011.

[6] U. Kiencke, Automotive control systems for engine, driveline, and vehicle, Springer, 2005.

[7] C. Guardiola, Detección y compensación de irregularidades de inyección a través de la medida del régimen instantáneo del turbogrupo, Reverté, 2005.

[8] Hillion, M., Chauvin, J., Grondin, O. y Petit, N., «Active combustion control of Diesel HCCI engine: Combustion timing,» SAE Technical Paper, 2008.

[9] Lapuerta, M., Armas, O. y Hernández, J. J., «Diagnosis of DI Diesel combustion from in-cylinder pressure signal by estimation of mean thermodynamic properties of the gas,» Applied Thermal Engineering 19, pp. 513-529, 1999.

[10] R. Moos, «A brief overview on automotive exhaust gas sensors based on electroceramics,» International Journal of Applied Ceramic Technology, 2(5), pp. 401-413, 2005.

[11] K. Ogata, Ingeniería de control moderna, Pearson Education, 2003.

[12] A. G. López, «Ecuaciones Diferenciales I,» Madrid, 2004, p. 115.

[13] Dauron, A., «Model-Based Powertrain Control: Many Uses, No Abuse,» Oil \& Gas Science and Technology, pp. 427-433, 2007. 
[14] J. E. Guarella, J. E. Guarella, L. Rodríguez y I. Bagatto, Sensores y actuadores en motores, Buenos Aires, 2011.

[15] Proakis, J. G. y Manolakis, D. G., Tratamiento digital de señales, Prentice Hall, 2007.

[16] BES - Bosch Extranet Service, Sistemas de Inyección Electrónica, 2010.

[17] Munoz, M. y Payri, F., Motores de combustión interna alternativos, Fundación General de la UPM, 1989.
[18] Galindo, J., Climent, H., Guardiola, C. y Doménech, J., «Modeling the vacuum circuit of a pneumatic vale system,» Journal of Dynamical Systems Measurement and Control, p. 131, 2009.

[19] D. C. Montgomery, Diseño y análisis de experimentos, Limusa-Wiley, 2002. 